\title{
Smartphone Ophthalmoscopy: is there a place for it?
}

\author{
Amar Pujari (iD \\ Dr Rajendra Prasad Centre for \\ Ophthalmic Sciences, All India Institute of \\ Medical Sciences (AlIMS), New Delhi, \\ India
}

\begin{abstract}
Smartphone technology is advancing at a rapid pace. Their role in day-to-day life is becoming more and more intricate and irreplaceable. Of late, they have gained immense importance in different medical specialities where they possess an active ability to guide the clinician. This is particularly evident in ophthalmology, where the constantly evolving camera-illumination systems and the artificial intelligence integrated technology have unravelled many novel observations for non-contact posterior segment imaging. The scope of this review is to highlight the role of smartphones as ophthalmoscopes (direct as well as indirect). Nevertheless, their limitations and future directions are also stated here with the intention of making progress in the field of smartphone fundus imaging.
\end{abstract}

Keywords: smartphones, smartphone ophthalmoscopy, smartphone fundoscopy

\section{Introduction}

Smartphones are the devices that have revolutionized our lives in 21 st century. The deeply integrated internet technology, evolving camera features and hopeful artificial intelligence interfaces have changed the very perspective of smartphone's role in medical subspecialities. ${ }^{1}$ Health care smartphone applications, tele-consultation from remote areas, and various other health monitoring features have changed the concept of health care from being mere physical observation to inseparable digital care. ${ }^{1}$ Hence, they have grown continuously and have proved their role as indispensable in some of the common sub-specialities. In ophthalmology, their usefulness is quite unique and growing. ${ }^{2,3}$ The ability to capture anterior as well as posterior segment images is an evidence of it. Previously, this author has enumerated various "smartphone imaging techniques" in ophthalmology ${ }^{2}$ and the "clinical role" in various posterior segment pathologies. ${ }^{3}$ While the future looks promising, the role of smartphones is currently limited to convenient screening devices rather than suitable quality diagnostic tools. This review summarises the data and opinions available in the medical literature to support the use of smartphones in ocular care as "smartphones ophthalmoscopes".

\section{Discussion}

\section{What is Smartphone Ophthalmoscopy and Why is It Necessary?}

As we know, the traditional direct and indirect ophthalmoscopy techniques have been in use clinically for many decades. At present, these tools are the gold standard for routine patient care as well as for teaching purposes. ${ }^{4,5}$ These
Correspondence: Amar Pujari

Email dramarpujari@gmail.com 
instruments have an inbuilt optical system, where a light source and an adjacent viewing set-up allow the effortless visualization of the optic nerve head and the surrounding retinal details. Even though these instruments are expensive, they are mandatory in routine ophthalmic practices. In addition, mastering these tools also requires a significant amount of practice and dedication, which every professional needs to exercise. ${ }^{4}$

Direct ophthalmoscopy is meant for targeted visualization, that is, a small area of the retina and the optic disc can be visualized. ${ }^{4}$ Unlike indirect ophthalmoscopy, it may not cover a larger area, but it enables magnified viewing of intended structures. On the other hand, indirect ophthalmoscopy is meant for a broader area of visualization, where it provides a larger field of view but minimizes the finer details. ${ }^{6}$ Thus, the two tools have different working principles and different clinical roles. In clinics, these two tools provide ample view of the fundus details needed to work and to diagnose most of the common clinical conditions.

However, the major limitation with these conventional ophthalmoscopes in the 21st century is the lack of digitalized features. Clinical photographs or the videos cannot be stored in, and cannot be retrieved from these devices, and more importantly they cannot be integrated with internet or artificial intelligence (AI) technology. Hence, to address these issues, a portable, non-contact and a universally accessible digital tool is highly desirable, where both direct and indirect ophthalmoscopic features can be combined with internet and AI technology.

Smartphones seem attractive and reliable tools, with the potential to fulfil all these aspirations. ${ }^{2,3}$ Their incredible photographic and video graphic features, and their working patterns are on par with many sophisticated tools, and most importantly they are very handy and pocket friendly.

In smartphones, the light source lies close to the camera but cannot be exactly simulated like a co-axial illumination system, which is very much necessary for fundus photography. Hence, by positioning an extra light source close to the inbuilt camera, desired pictures or videos can be obtained. Furthermore, by adjusting the camera and the lens type, small angle, wide angled and ultrawide angle pictures can be obtained. This is smartphone ophthalmoscopy, which is now gaining rapid acceptance because of its many advantages and its ability to mould with the current as well as future technologies.

\section{Smartphones as Direct Ophthalmoscopes}

Using smartphones as direct ophthalmoscopes, innovators have tried and developed various clip attachments. These clips, after assembling with the smartphones, allow necessary visualization of the optic disc and the retinal pathologies, hence, this co-ordinated tool can be used as direct ophthalmoscope.

For this purpose, the attachments are evolving, and to name few, "D eye", "Peek", "Cell-scope" and others have proven their role in different study set-ups (the author has no financial interests). ${ }^{2,3,7}$ Clinicians and health care workers who are interested in documenting optic disc and the macular findings can try any of these attachments and can document desired images electronically. The optic disc cupping, neuro-retinal rim health, optic disc vasculature, retinal nerve fibre layer health, foveal details, papillomacular bundle health and other relevant information can be captured and stored for future references. ${ }^{8-12}$ The captured area may be variable from device to device, but they can surely capture the disc and the macular details depending upon the need. Nevertheless, during image capturing, the distance between the smartphone and the eye, smartphone inclination (so as to avoid the light reflexes from the corneal surface), image brightness, and other visually important parameters must be taken into consideration to achieve clear and well-focussed images. If the above parameters are set incorrectly, then the clinical interpretation can be skewed and the diagnosis can be incorrect.

Similarly, during smartphone direct-ophthalmoscopic examination, patient comfort is also equally important; the brightness of the light source, dazzle reflex, pupil size and the anterior media clarity are crucial for optimal photography. Nevertheless, the expertise and the amount of time spent on perfecting these techniques is also equally important. During initial attempts, invariably the patient as well as the examiners are likely to experience some difficulty unlike smartphone photography in routine life. Focusing through the pupil, searching for the optic disc, and stabilization of the eye are all important. Therefore, smartphone direct ophthalmoscopy is a good concept, but before concluding their strengths and limitations, optimal exploration and understandings are necessary.

Another limitation associated with smartphone direct ophthalmoscopy is that unlike traditional direct ophthalmoscopes, different apertures of light source such as streak light, fixation target and filters are absent here. Therefore, in future, if smartphone ophthalmoscopy gains wider 
acceptance, then these features can be integrated through added applications to improve overall quality.

Nevertheless, to make smartphone direct ophthalmoscopy a more interesting concept, the author is of the opinion that it needs to be without any attachments. This is because, often people forget the attachments, or they do not carry them everywhere like smartphones. Therefore, to fulfil this lacuna, Gunasekera and Thomas description of iPhone $\mathrm{X}$ alone seems interesting, ${ }^{13}$ and should be explored further. ${ }^{14}$ The current author has shown that using iPhone $\mathrm{X}$ alone, wider and clearer photographs of the optic disc and the macula can be captured, ${ }^{14}$ however, here also a learning curve exists, and this technique also needs some extra patience. Hence, in future, if our handy smartphones can act as direct ophthalmoscopes with no or minimal attachments, then the clinicians are likely to show keen interest in using them for routine care. ${ }^{15,16}$

\section{Smartphones as Indirect/Wide Angled Ophthalmoscopes}

The concept here is not necessarily a binocular visualization as we have seen it on indirect ophthalmoscopy, but it intends to acquire a wider field of view like that of an indirect ophthalmoscope. Using different lenses, an image spanning from standard photograph to wide-angled retinal pictures can be acquired, ${ }^{17}$ and unlike the head-band assisted indirect ophthalmoscopes or the fixed imaging devices (fundus camera and other montage/wider imaging tools), just using the smartphones with an attachment the wide-angled retinal picture can be captured. The following points highlight the overall concept of wide angled retinal imaging using smartphones.

Firstly, by holding a routine 20 dioptre lens and the smartphone in a co-axial fashion (using the video mode with continuous light source on) images equivalent to that of an indirect ophthalmoscopy can be captured. This usually documents fundus images from superior retinal arcade to inferior retinal arcade in one frame. ${ }^{18}$ Nevertheless, two separate plano-convex lenses 10 $\mathrm{D}$ each can also be used, to acquire either standard fundus pictures (20D) or magnified macular pictures (10D).

Secondly, other increasingly wider lenses can also be used to acquire images well beyond the equator, and even up to the periphery, simulating a wide angled retinal photograph. ${ }^{19,20}$ The available range of lenses here is immense, with each having different fields of capture and variable configuration. ${ }^{17}$
Thus, cumulatively, smartphone fundus photography can be effectively used for screening purposes (including macular pathologies, diabetic retinopathy, retinopathy of pre-maturity, retinoblastoma and others). This specially aids in decentralising multi-speciality health care in developing countries. ${ }^{21-31}$

\section{General Ophthalmologist's Expectations from Smartphone Ophthalmoscopic Studies}

As every general ophthalmologists and dedicated posterior segment practitioners buy an ophthalmoscope and fundus photographic machine for their routine clinical practices, the first question they ask is whether they really need these smartphone tools or are they missing some of the technological advancements in their area? At this juncture, their concerns are genuine and valid, as we are aware, the smartphone observations in ophthalmology are evolving and significant progress has been achieved in the past ten years, where many young innovators have come up with their own ideas and have devised many useful devices. Hence, as of now, based on literature evidences, the practitioners or enthusiasts can consider these tools for screening purposes or for quick image captures where resources are deficient.

Second, some beginners often ask can these tools provide fundus pictures, which are on par with or superior to routine fundus tools? To this, we all know that the fundus cameras have established their role over a period of time, the initial fundus camera images were not on par with the current imaging techniques, the things have made significant progress since their inception, and hence we now consider them as gold standard tools for regular examination, follow ups, and for teaching purposes and for publications. As of now, smartphones do provide reasonable quality of photographs, which can be considered on par with fundus camera pictures. However, the replacement of fundus camera images with smartphone camera images is yet to be answered.

Third, the various devices have created confusion among users because of their variable size, their price, and amount of area captured. Again, a homogeneous consensus in these areas is lacking; hence, future constructive efforts are needed in the years to come to reach more reasonable conclusions. Nevertheless, the user must read the full functionality of the devices before purchasing them. 


\section{Practical Solutions for Fundus Imaging Using Smartphone Ophthalmoscopes}

Whenever the author presented his work and other defined techniques from the literature, the peers and/or the smartphone enthusiasts frequently asked for a simpler, reliable and cheaper alternative. In fact, their expectation was that the smartphone imaging should be as simple as taking normal photographs. Which in fact is very difficult at this point of time. They need to understand that we have begun the journey just now, and many difficulties are still unaddressed; therefore, to optimise their photography skills, an understanding on following key points is must.

1. Whenever a naïve colleague starts using smartphone ophthalmoscopy for imaging, understanding how exactly the device works is important.

2. Then, they must decide their area of interest, that is whether they want to image the optic disc or the retina, and if retina, whether they want a regular field of view or a wider field of view. And then they must set that mode.

3. Next, to capture the intended tissue of interest and the area, optimal positioning of the device from eye, ways to stabilize their hands and ways to achieve desired inclination (to avoid reflexes) must be learnt beforehand.

4. Then, ways to achieve better illumination, patient comfort, tricks to avoid glare, desired pupil size and measures to counter eye movements must be learnt and mastered with time.

5. Last but not the least, persistence, patience and dedication are vital to make future progresses.

\section{Where are We in the Journey of Smartphone Ophthalmoscopy}

In the journey of smartphone ophthalmoscopes, we are in the beginning phase, where different possibilities of digital documentation are being searched for. The gathered understandings are progressing and more importantly everyone is aware of what needs to be achieved. That is, easy and effortless documentation of ocular images on smartphones with a quick diagnosis and a management plan using artificial intelligence technology. ${ }^{32}$

\section{Conclusions}

To conclude, the question is "smartphone ophthalmoscopy: is there a place for it?" And the answer is in two parts. Yes, they have a practical role for preliminary or screening or learning purposes, and No or not yet clear for routine ophthalmic practices. The general ophthalmologists must choose their devices wisely based on their needs and the area of interest, and then, all-inclusive efforts must be made to master them.

\section{Disclosure}

The author reports no conflicts of interest in this work.

\section{References}

1. Ozdalga E, Ozdalga A, Ahuja N. The smartphone in medicine: a review of current and potential use among physicians and students. J Med Internet Res. 2012;14(5):e128. doi:10.2196/ jmir.1994

2. Pujari A, Saluja G, Agarwal D, Selvan H, Sharma N. Clinically useful smartphone ophthalmic imaging techniques. Graefes Arch Clin Exp Ophthalmol. 2021;259(2):279-287. doi:10.1007/s00417020-04917-z

3. Pujari A, Saluja G, Agarwal D, et al. Clinical role of smartphone fundus imaging in diabetic retinopathy and other neuro-retinal diseases. Curr Eye Res. 2021;46(11):1605-1613. doi:10.1080/ 02713683.2021.1958347.

4. Benbassat J, Polak BC, Javitt JC. Objectives of teaching direct ophthalmoscopy to medical students. Acta Ophthalmol. 2012;90 (6):503-507. doi:10.1111/j.1755-3768.2011.02221.x

5. Yusuf I, Salmon J, Patel C. Direct ophthalmoscopy should be taught to undergraduate medical students-yes. Eye. 2015;29:987-989. doi:10.1038/eye. 2015.90

6. Roybal CN. Indirect Ophthalmoscopy 101; 2017. Available from: https://www.aao.org/young-ophthalmologists/yo-info/article/indirectophthalmoscopy-101. Accessed October 21, 2021.

7. Ludwig CA, Murthy SI, Pappuru RR, et al. A novel smartphone ophthalmic imaging adapter: user feasibility studies in Hyderabad, India. Indian J Ophthalmol. 2016;64(3):191-200. doi:10.4103/03014738.181742

8. LaMonica LC, Bhardwaj MK, Hawley NL, et al. Remote screening for optic nerve cupping using smartphone-based nonmydriatic fundus photography. $J$ Glaucoma. 2021;30(1):58-60. doi:10.1097/ IJG.0000000000001680

9. Giardini ME, Livingstone IAT, Jordan S, et al. A smartphone based ophthalmoscope. In 2014 36th Annual International Conference of the IEEE Engineering in Medicine and Biology Society; 2014:2177-2180.

10. Bastawrous A, Giardini ME, Bolster NM, et al. Clinical validation of a smartphone-based adapter for optic disc imaging in Kenya. JAMA Ophthalmol. 2016;134(2):151-158. doi:10.1001/ jamaophthalmol.2015.4625

11. Wintergerst MWM, Brinkmann CK, Holz FG, Finger RP. Undilated versus dilated monoscopic smartphone-based fundus photography for optic nerve head evaluation. Sci Rep. 2018;8(1):10228. doi:10.1038/ s41598-018-28585-6

12. Pujari A, Mukhija R, Chawla R, Phuljhele S, Saxena R, Sharma P. Smartphone-based evaluation of the optic nerve head. Indian $J \quad$ Ophthalmol. 2018;66(11):1617-1618. doi:10.4103/ijo. IJO_394_18

13. Gunasekera CD, Thomas P. High-resolution direct ophthalmoscopy with an unmodified iPhone X. JAMA Ophthalmol. 2019;137 (2):212-213. doi:10.1001/jamaophthalmol.2018.5806

14. Pujari A, Markan A, Chawla R, Gagrani M. The additional role of unmodified iPhone $\mathrm{X}$ as a direct ophthalmoscope. Indian J Ophthalmol. 2019;67(7):1253-1254. doi:10.4103/ijo.IJO_90_19 
15. Pujari A, Selvan H, Goel S, Dada T. Direct smartphone disc video documentation for pediatric glaucomas during evaluation under anesthesia. J Glaucoma. 2019;28(8):e143-e144. doi:10.1097/ IJG.0000000000001294

16. Pujari A, Selvan H, Goel S, Ayyadurai N, Dada T. Smartphone disc photography versus standard stereoscopic disc photography as a teaching tool. J Glaucoma. 2019;28(7):e109-e111. doi:10.1097/ IJG.0000000000001251

17. Rajalakshmi R, Prathiba V, Arulmalar S, Usha M. Review of retinal cameras for global coverage of diabetic retinopathy screening. Eye. 2021;35(1):162-172. doi:10.1038/s41433-020-01262-7

18. Bastawrous A. Smartphone fundoscopy. Ophthalmology. 2012;119 (2):432-433.e2; author reply 433. doi:10.1016/j.ophtha.2011.11.014

19. Maamari RN, Keenan JD, Fletcher DA, Margolis TP. A mobile phone-based retinal camera for portable wide field imaging. $\mathrm{Br}$ J Ophthalmol. 2014;98(4):438-441. doi:10.1136/bjophthalmol-2013303797

20. Kim TN, Myers F, Reber C, et al. A smartphone-based tool for rapid, portable, and automated wide-field retinal imaging. Transl Vis Sci Technol. 2018;7(5):21. doi:10.1167/tvst.7.5.21

21. Tan CH, Quah WH, Tan CSH, Smith H, Tudor Car L. Use of smartphones for detecting diabetic retinopathy: a protocol for a scoping review of diagnostic test accuracy studies. BMJ Open. 2019;9(12):e028811. doi:10.1136/bmjopen-2018-028811

22. Queiroz MS, de Carvalho JX, Bortoto SF, et al. Diabetic retinopathy screening in urban primary care setting with a handheld smartphone-based retinal camera. Acta Diabetol. 2020;57 (12):1493-1499. doi:10.1007/s00592-020-01585-7

23. Gulshan V, Peng L, Coram M, et al. Development and validation of a deep learning algorithm for detection of diabetic retinopathy in retinal fundus photographs. JAMA. 2016;316(22):2402-2410. doi:10.1001/jama.2016.17216

24. Natarajan S, Jain A, Krishnan R, Rogye A, Sivaprasad S. Diagnostic accuracy of community-based diabetic retinopathy screening with an offline artificial intelligence system on a smartphone. JAMA Ophthalmol. 2019;137(10):1182. doi:10.1001/jamaophthalmol.2019. 2923
25. Rajalakshmi R, Subashini R, Anjana RM, Mohan V. Automated diabetic retinopathy detection in smartphone-based fundus photography using artificial intelligence. Eye Lond Engl. 2018;32 (6):1138-1144

26. Malerbi FK, Andrade RE, Morales PH, et al. Diabetic retinopathy screening using artificial intelligence and handheld smartphone-based retinal camera. J Diabetes Sci Technol. 2021:1932296820985567. doi:10.1177/1932296820985567

27. Hwang DK, Yu WK, Lin TC, et al. Smartphone-based diabetic macula edema screening with an offline artificial intelligence. J Chin Med Assoc JCMA. 2020;83(12):1102-1106. doi:10.1097/ JCMA.0000000000000355

28. Valikodath N, Cole E, Chiang MF, Campbell JP, Chan RVP. Imaging in retinopathy of prematurity. Asia-Pac J Ophthalmol. 2019;8 (2):178-186.

29. Wintergerst MWM, Petrak M, Li JQ, et al. Non-contact smartphone-based fundus imaging compared to conventional fundus imaging: a low-cost alternative for retinopathy of prematurity screening and documentation. Sci Rep. 2019;9(1):19711. doi:10.1038/ s41598-019-56155-x

30. Patel TP, Kim TN, Yu G, et al. Smartphone-based, rapid, wide-field fundus photography for diagnosis of pediatric retinal diseases. Transl Vis Sci Technol. 2019;8(3):29. doi:10.1167/tvst.8.3.29

31. Pujari A, Lomi N, Goel S, et al. Unmodified iPhone XS Max for fundus montage imaging in cases of retinoblastoma. Indian J Ophthalmol. 2019;67(6):948-949. doi:10.4103/ijo.IJO_2144_18

32. Wintergerst MW, Jansen LG, Holz FG, Finger RP. Smartphone-based fundus imaging-where are we now? Asia-Pac J Ophthalmol. 2020;9 (4):308-314. doi:10.1097/APO.0000000000000303
Clinical Ophthalmology

\section{Publish your work in this journal}

Clinical Ophthalmology is an international, peer-reviewed journal covering all subspecialties within ophthalmology. Key topics include: Optometry; Visual science; Pharmacology and drug therapy in eye diseases; Basic Sciences; Primary and Secondary eye care; Patient Safety and Quality of Care Improvements. This journal is indexed on PubMed
Dovepress

Central and CAS, and is the official journal of The Society of Clinical Ophthalmology (SCO). The manuscript management system is completely online and includes a very quick and fair peer-review system, which is all easy to use. Visit http://www.dovepress.com/ testimonials.php to read real quotes from published authors. 